\title{
Study the Flow behind a Semi-Circular Step Cylinder (Laser Doppler Velocimetry (LDV) and Computational Fluid Dynamics (CFD))
}

\section{S. M. Sayeed-Bin-Asad *, Tord Staffan Lundström and Anders Gustav Andersson}

Division of Fluid and Experimental Mechanics, Luleå University of Technology, SE-971 87 Luleå, Sweden; staffan.lundstrom@ltu.se (T.S.L.); anders.g.andersson@ltu.se (A.G.A.)

* Correspondence: sayeed.asad@ltu.se; Tel.: +46-920-492-886

Academic Editor: Bjørn H. Hjertager

Received: 1 February 2017; Accepted: 6 March 2017; Published: 9 March 2017

\begin{abstract}
Laser Doppler Velocimetry (LDV) measurements, flow visualizations and unsteady Reynolds-Averaged Navier-Stokes (RANS) Computational Fluid Dynamics (CFD) simulations have been carried out to study the turbulent wake that is formed behind a semi-circular step cylinder at a constant flow rate. The semi-circular cylinder has two diameters, a so-called step cylinder. The results from the LDV measurements indicate that wake length and vortex shedding frequency varies with the cylinder diameter. This implies that a step cylinder can be used to attract fish of different size. By visualizations of the formation of a recirculation region and the well-known von Kármán vortex street behind the cylinder are disclosed. The simulation results predict the wake length and shedding frequency well for the flow behind the large cylinder but fail to capture the dynamics of the flow near the step in diameter to some extent and the flow behind the small cylinder to a larger extent when compared with measurements.
\end{abstract}

Keywords: laser doppler velocimetry (LDV); computational fluid dynamics (CFD); fish migration; step cylinder; shedding; vortex street

\section{Introduction}

Hydro and nuclear power are the major sources of electricity in Sweden. Swedish Energy [1] has recently published some statistics regarding power production in Sweden showing that hydro and nuclear power stand for $47 \%$ and $34 \%$ of the total electricity in Sweden, respectively. In order to increase the percentage renewable energy overall, to keep the target of greenhouse gas emission [2], systems are constantly developed to harvest wind, wave, tidal, and solar energy. These sources of energy are typically irregular and continuous power production is required to cover for this irregularity. The availability, storage capacity, and flexibility of hydropower make it a perfect contender for the power regulation role. However, hydropower has some local impacts on the environment, such as creating dams in rivers and changing water flow directions, which lead to some problems for fauna passage, in general, and migrating fishes, in particular. Fish migration issues are mostly studied from a biological point of view and more detailed studies from a fundamental fluid mechanics point of view could lead to new insights and solutions.

Some hydropower dams have fishways or fish ladders to allow fish to migrate past dams, as well as past rotating turbines and, during swimming or passing this fishway or fish ladder, fish have to tackle different flow obstructions or disturbances, like turbine intakes, stones, and concrete structures. Fluid flow characteristics in fish ladders or fishways during fish migration are crucial when designing effective fishways so that fishes migrate in an efficient way. Studies [3-7] have shown that cylindrical obstructions can be used to create favorable conditions for upstream migrating fishes of 
appropriate size. The concept is to create periodic vortices that the fish uses in an energy efficient way (less energy than in a free stream) to maintain a position in the flow. Liao [8] describes the structure of the flow around a half cylinder and the positions of fish swimming within the wake behind and around the cylinder. It was found that the fishes exploit the flow conditions created by the semicircular cylinder when swimming upstream around it. Apart from this, Liao discovered the altered shedding frequencies and wakes for various cylinder diameters. Different sizes of fish exploit vortices with different shedding frequencies. Therefore, it is important to have proper shedding for appropriate sizes of fish to swim past obstructions.

There are many applications for step full cylinders, including airport control towers, street lamp posts, antenna members and radio communication towers. Hence, there are several driving forces for engineers to examine this type of flow phenomena, both numerically and experimentally.

Dunn et al. [9] carried out velocity measurement with laser doppler velocimetry (LDV) and flow visualization around a step cylinder. One result is that the recirculation near a step has significant effects on the shedding frequencies as compared to locations away from the step. Similarly, another study from Dunn et al. [10] also found significant effects of free-stream shearing on vortex-shedding characteristics from a step-cylinder. Chris Morton et al. [11-16] carried out several studies using both numerical and experimental with 2D and 3D particle image velocimetry (PIV) investigations on step cylinders, such as single step dual step, etc. Detailed studies of the wake vortex dynamics behind step-cylinder are provided. He [17] has recently used tomographic-PIV (3D-PIV) behind a step cylinder for three-dimensional (3D) information of vortex shedding. Rafati [18] conducted a series of experimental studies with various diameters of step cylinders using tomographic-PIV measurement. One result is that for high flow (higher velocity than free stream velocity), streamwise vortices are introduced into the wake that complicates the vortex dynamic, and vortex interactions. Rafati [18] also produced a table of studies on step cylinders available (Table 1).

Table 1. Previous studies on flow behind step cylinders. Reproduced from [18].

\begin{tabular}{ccc}
\hline Study & $\boldsymbol{R} \boldsymbol{e}_{\boldsymbol{D}}$ & $\boldsymbol{D} / \boldsymbol{d}$ \\
\hline N.Ko et al. [19] & $8 \times 10^{4}$ & 2 \\
Yagita et al. [20] & $8 \times 10^{2}-10^{4}$ & $1.25-5$ \\
Lewis et al. [21] & $67-200$ & $1.14-1.76$ \\
Dunn et al. [10] & $62-1230$ & 2 \\
Norberg [22] & $3 \times 10^{3}-13 \times 10^{3}$ & $1.25-2$ \\
Chua et al. [26] & $8 \times 10^{4}$ & $1.33-2.78$ \\
Morton et al. [11,27,28] & $4.72 \times 10^{3}$ & 3 \\
N.Ko et al. (1984, 1990, 1992) [23-25] & $150-2100$ & 2 \\
Dunn et al. [9] (span-wise sheared flow) & $152-764$ & 1.92 \\
\hline
\end{tabular}

Despite having some investigations of flow behavior around stepped cylinders, little or no scientific works, especially experimental, on half cylinders with steps has been performed. Therefore, in the current study the flow around such an object is studied with LDV and computational fluid dynamics (CFD). The flow dynamics of the step and diameter change is interesting from a fluid mechanics point of view but as the half cylinders also have shown to be interesting for fish migration, the difference in wake formation and shedding of vortices behind the different cylinder diameters could also be designed to fit different sizes and species of fish.

\section{Experimental Arrangement}

Experiments were carried out in a water flume at the John Field Laboratory at Luleå University of Technology, Sweden. The flume is $7.5 \mathrm{~m}$ long with a cross-section of $0.295 \mathrm{~m} \times 0.310 \mathrm{~m}$. The semi-circular step cylinder employed in this investigation is made of acetal (POM) and the diameter of the small $(d)$ and large $(D)$ cylinders are $50 \mathrm{~mm}$ and $100 \mathrm{~mm}$, respectively. Figure 1 describes the configuration and measuring zones of the cylinder centerline. 


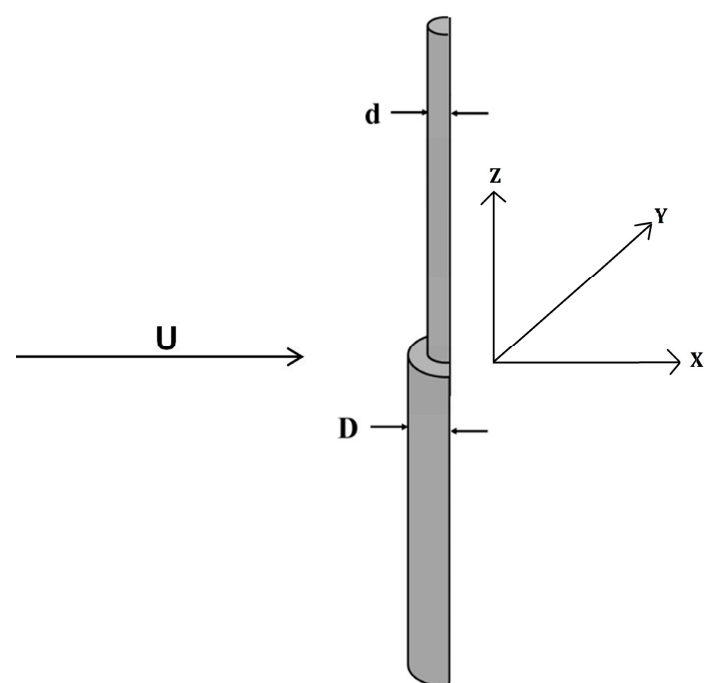

(a)

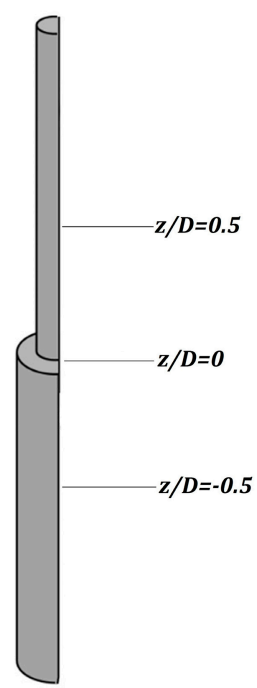

(b)

Figure 1. Cylinder configuration; (a) flow direction and coordinate description; and (b) measuring zones.

A submersible pump was used to pump the water through the channel from a rectangle water reservoir tank with $2 \mathrm{~m}^{3}$ storage capacity. The water reservoir is placed at the outlet of the channel and the temperature of the water was controlled using an automatic cooling arrangement. This is essential since variation in water temperature changes its viscosity and refractive index properties. Changes in water temperature leads to laser beam displacements from the desired point of measurements. A Danfoss MassFlo Corolios flow meter was used to control the water flow rate.

To obtain a uniform velocity distribution through the channel there is a steel net and a honeycomb at the inlet of the channel. The thickness of the honeycomb is $75 \mathrm{~mm}$ and the diameter of its wholes is $7.6 \mathrm{~mm}$. The steel net geometry consists of $2.5 \mathrm{~mm} \times 2.5 \mathrm{~mm}$ square holes and it is $0.8 \mathrm{~mm}$ thick. A schematic arrangement of the experimental set-up is presented Figure 2.

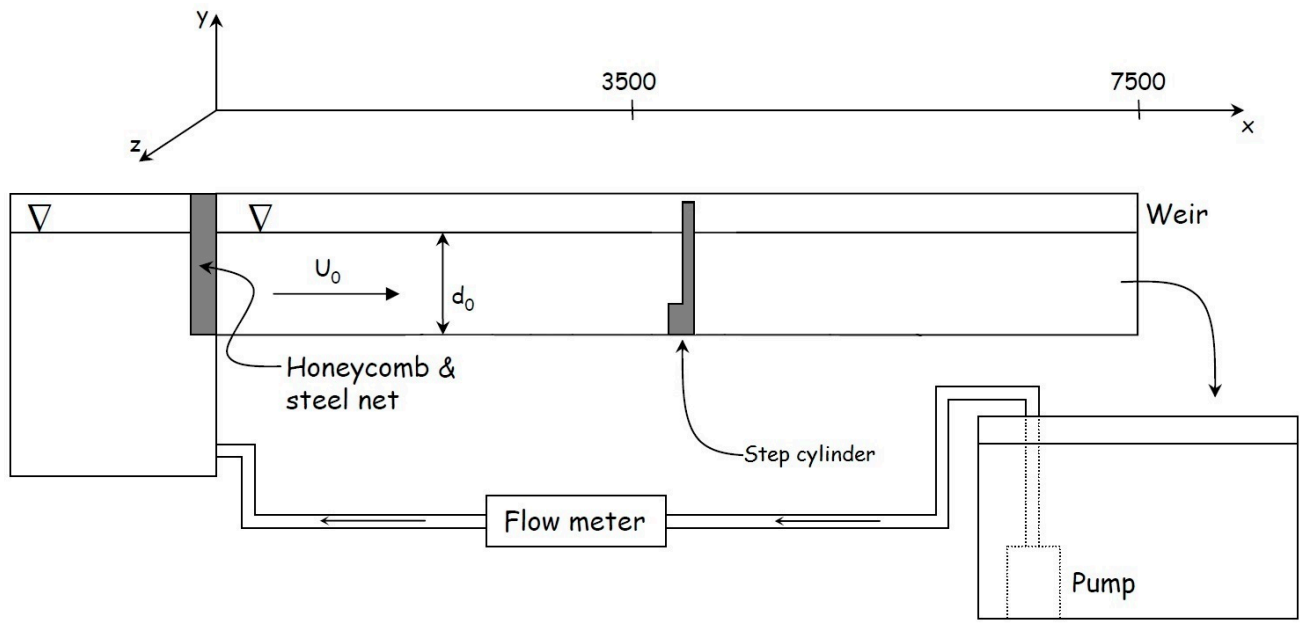

Figure 2. Schematic presentation of water circulation in the flume.

This LDV system, used in this investigation, is a two component configuration with an $83 \mathrm{~mm}$ diameter optical fiber probe and a front lens with $360 \mathrm{~mm}$ focal length which consists of a $300 \mathrm{~mW}$ diode-pumped solid-state laser, and transmitting optics, including a beam splitter Bragg-cell, photo detector, and signal processor. The system is used in backscatter mode so that the probe is both 
transmitting and receiving signals. The laser has two channels with a wavelength of 532 and $561 \mathrm{~nm}$, respectively. The laser power is $300 \mathrm{~mW}$ for each channel. The smallest dimensions of the measurement volume are approximately $0.123 \times 0.117 \mathrm{~mm}^{2}$ for both colors, and the system is operated in coincidence mode, which ensures correlated velocity components. The water was seeded with polyamide particles with a diameter of $5 \mu \mathrm{m}$ (Dantec's PSP-5). TSI's FlowSizer 64 software (Shoreview, MN, USA) was used for the data acquisition and data acquisition rates were tried to maintain greater than $50 \mathrm{~Hz}$. The 2D-LDV probe was mounted on a 3D traversing mechanism (can travel maximum $600 \mathrm{~mm}$ in each direction) controlled by the software. The sampling time was maintained as 121 seconds at each measurement point, corresponding to at least 10,000 samples, which are essential for statistical precision. All of the measurements were carried out in coincidence mode [29] to obtain an equal number of velocity counts for each direction, and the free stream velocity $\left(U_{\infty}\right)$ was determined from repeated measurements at a position $155 \mathrm{~mm}$ upstream of the cylinder at $z / D=0$ as Norberg [30] determined. Free stream $\left(U_{\infty}\right)$ is also calculated theoretically using the flow rate $\left(0.0077 \mathrm{~m}^{3} \cdot \mathrm{s}^{-1}\right)$ and it is found to be $U_{\infty}=0.1451 \mathrm{~m} \cdot \mathrm{s}^{-1}$. Repeatability tests are completed to estimate the first-order variable uncertainty for the experiments [31]. Four different measurements for all experiments were completed to estimate the random error introduced by the experimental facility and changes in experimental conditions, such as the water temperature, position of the cylinder, etc.

Flow visualization was also conducted to explore the wake structure using a digital single-lens reflex (DSLR) camera (Nikon D90) and two tungsten light heads to illuminate the flow area. Two food dyes (red and green) were injected from both sides of the cylinder using a programmable syringe pump running at constant speed. Videos were recorded from both the side and top of the flume and the recordings were converted into images in equal time step using MATLAB (The MathWorks, Natick, MA, USA).

Except otherwise mentioned for scaling the results, all positions and distances are scaled with $D$ and velocities with the free stream velocity $U_{\infty}$.

\section{Numerical Setup}

The simulations were performed with the commercial software Ansys CFX 16 (ANSYS, Inc, Canonsburg, PA, USA). A structured grid with 10.1 million nodes and an average wall resolution of $y^{+}=0.7$ and maximum $y^{+}=2.8$ was created in ANSYS IcemCFD (275 Technology Drive, Canonsburg, PA, USA). The volume of fluids (VOF) method was used to account for both the water and air phase. The numerical domain did not include the full flume, the inlet was placed $1 \mathrm{~m}$ upstream the cylinder and the outlet was placed $1.5 \mathrm{~m}$ downstream to reduce computational time. To generate a fully developed inlet profile an additional simulation model was created for the upstream part of the flume with a constant velocity inlet assumed at the honeycomb and the velocity profile from the outlet of that simulation was used as inflow for the domain with the cylinder. A second-order accurate advection scheme was used to solve the flow equations and a second-order backward Euler transient scheme was used for the temporal discretization. A timestep of $0.025 \mathrm{~s}$ was selected which corresponds to a RMS Courant number of $\sim 5$ and the relevant transient results were saved every 10 time steps. The streamwise velocity component was monitored at 16 points in the central plane on three different heights corresponding to the measurement positions from the LDV. The shear stress transport (SST) turbulence model was selected [32] as it utilizes the near-wall capabilities of the k- $\omega$ model and at the same time uses the bulk flow from the k- $\varepsilon$ model where the $k-\omega$ is weaker. The flow was initialized with a constant velocity corresponding to the measured mass flow $\left(0.0077 \mathrm{~m}^{3} \cdot \mathrm{s}^{-1}\right)$. After an initial settling period of $\sim 1000$ iterations the flow became more periodic and a developed vortex shedding was assumed. The simulation data was then saved for 2500 iterations or $62.5 \mathrm{~s}$ resulting in a total computational time of $\sim 4$ weeks on 64 CPU cores. 


\section{Results and Discussion}

Migrating fishes swimming around cylindrical obstructions face vortices and, interestingly, these vortices provide extra energy to fishes to improve their swimming abilities. These vortices are influenced by wake and shedding frequency, but these wake and vortex shedding frequencies are greatly dependent on the cylinder diameter [8]. A cylinder with different diameters, such as a step cylinder, could generate an interesting flow behind it and variable wake and shedding frequencies. This warrants this study of wake and vortex shedding behind a semicircular step cylinder with LDV and CFD.

\subsection{Wake}

Time-averaged measurements of centerline velocity distribution behind the step cylinder at $z / D=-0.5, z / D=0.0$, and $z / D=0.5$ are shown in Figure $3 a-c$ and notice that for all positions the negative values captured indicate a wake. A main result is that behind the step $(z / D=0.0)$, the wake is longer compared to the situation behind both the large and small cylinders (see Figure $3 a-c$ and Table 2). The simulations, on the other hand, yield that the length of the wake behind the large cylinder $(z / D=-0.5)$ is shorter than for the other cases and the time averaged local velocity $\bar{u}$ swiftly goes back toward free stream velocity, $U_{\infty}$. There is, therefore, good agreement between the simulation and experiments (see Figure 3a). The agreement is also fairly good between simulations and measurements at the step while the simulations severely over predict the length of the wake behind the small cylinder $(z / D=0.5)$ and the simulated results never approach the free stream velocity in the interval of measurement (see Figure 3c) and Table 2 also yields that although simulations compare well to experiments behind the large cylinder and at the step, the simulations always overpredict the length of the wake.

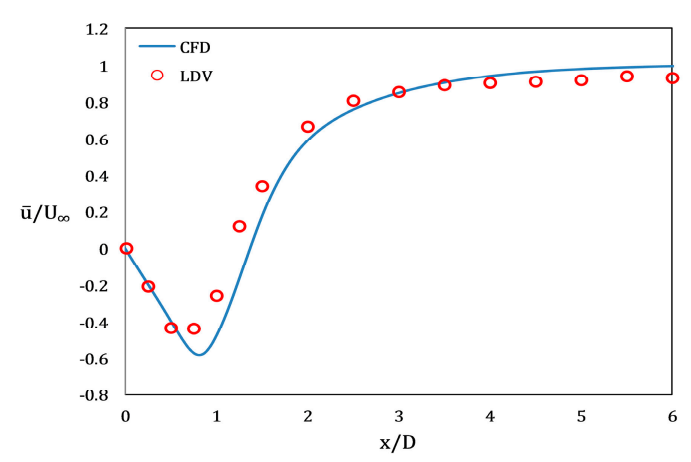

(a)

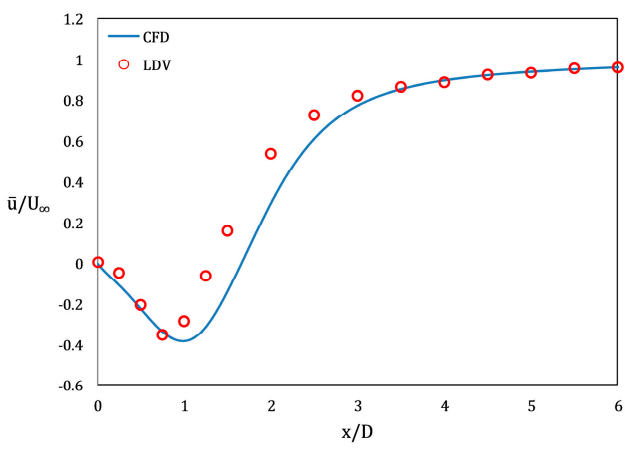

(b)

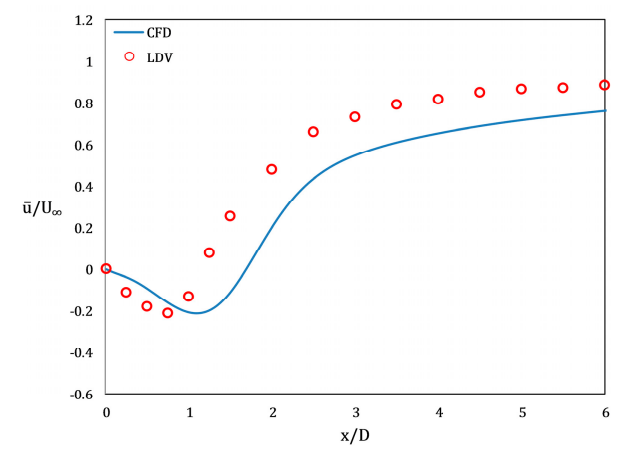

(c)

Figure 3. Wake centerline velocity at (a) $z / D=-0.5 ;(\mathbf{b}) z / D=0.0 ;$ and (c) $z / D=0.5$. 
Table 2. Wake length behind the step cylinder.

\begin{tabular}{ccccc}
\hline Description & Position $(z / D)$ & Case & Position $(x / D)$ & Wake Length $(\mathbf{m m})$ \\
\hline \multirow{2}{*}{ Downward 50 mm } & \multirow{2}{*}{0.5} & CFD & 1.4 & 139 \\
& & LDV & 1.25 & 125 \\
\hline \multirow{2}{*}{ Along step } & \multirow{2}{*}{0} & CFD & 1.7 & 169 \\
& & LDV & 1.5 & 150 \\
\hline \multirow{2}{*}{ Upward 50 mm } & \multirow{2}{*}{0.5} & CFD & 1.76 & 176 \\
& & LDV & 1.25 & 125 \\
\hline
\end{tabular}

The wake behind the cylinder can be qualitatively visualized by the average of streamwise velocity as derived in the simulations, as seen in Figure 4. The wake is symmetrical in the horizontal plane (Figure 4a) which suggests that the velocity field was averaged for a sufficiently long time; the bow wake [7] in front of the cylinder is also captured. When scrutinizing the vertical plane (Figure 4b) the wake is longer down-stream the smaller cylinder. It can also be seen that within the wake behind the step cylinder a recirculation region develops immediately after the cylinder where reverse flow $(\bar{u}<0)$ is observed (Figures 3 and 4), which extends roughly one diameter downstream of the cylinder. Mean velocity, $\bar{u}$ rapidly approaches the free stream velocity $U_{\infty}$ after the recirculation region. The velocity after the wake is not capable of recovering completely as the drag-induced momentum deficit must remain constant behind the cylinder (see Figure 3). The continuum is, instead, reserved by a slight increase in the level of the surface.

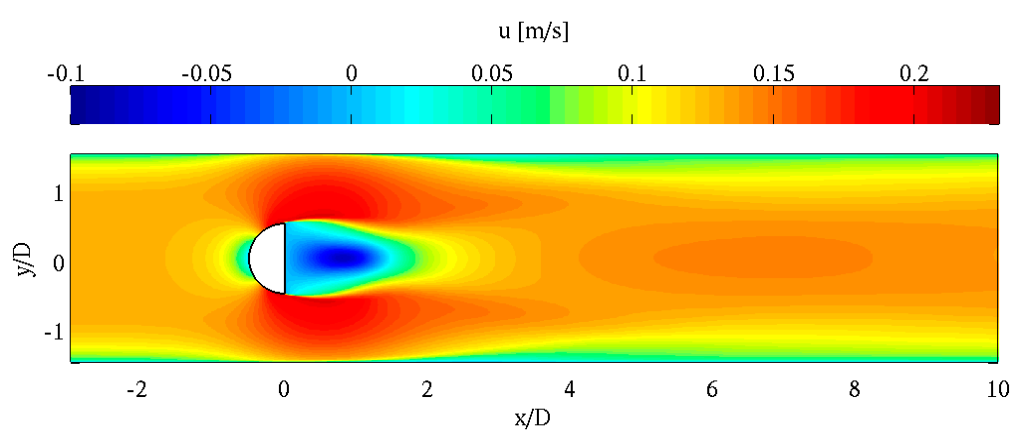

(a)

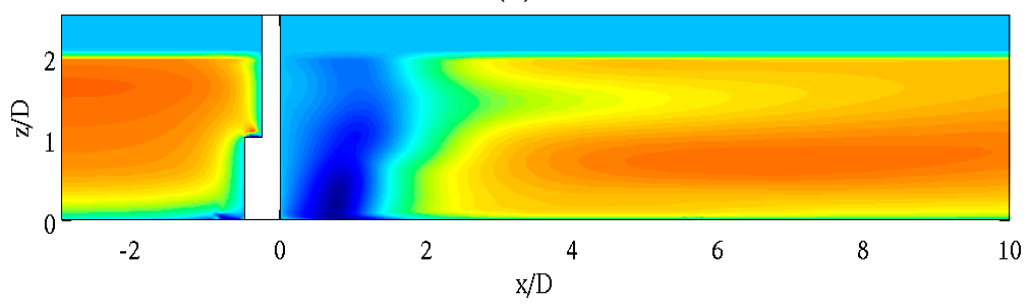

(b)

Figure 4. Averaged streamwise velocity at (a) $z / D=-0.5$; and (b) $y / D=0.0$.

The wake structures have also been visually observed from the side of a semi-circular cylinder with a step. For instance, the structures of a wake were found behind the step cylinder at $z / D=-0.5$ with a $1 \mathrm{~s}$ interval $(t, t+1$ and $t+1+1)$ are presented in Figure $5 \mathrm{a}-\mathrm{c}$, respectively. Dyes (red and green) were injected from the both sides of the step cylinder. It is seen that the dyes were swept into the well-known von-Karman vortex street right after downstream of the cylinder. Additionally, three-dimensional (3D) flow structures were also observed behind the cylinder, and a mainly downward flow was noticed. 


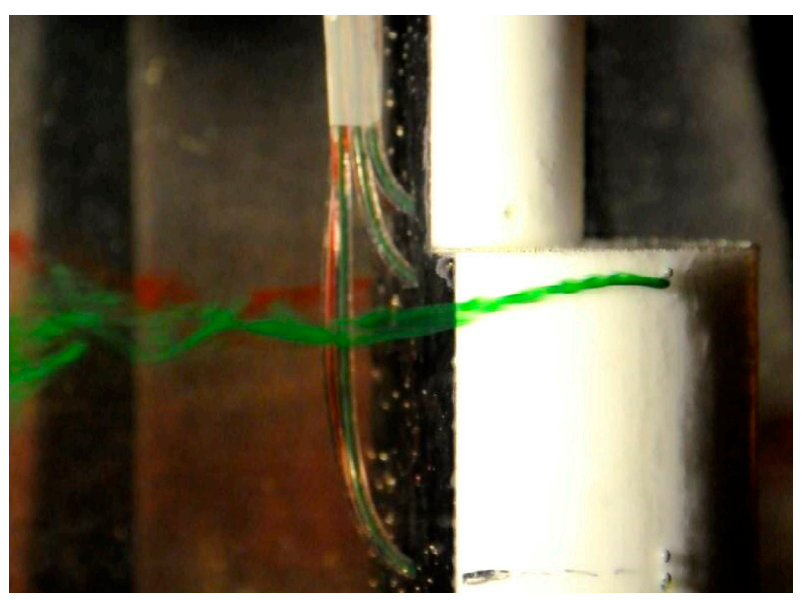

(a)

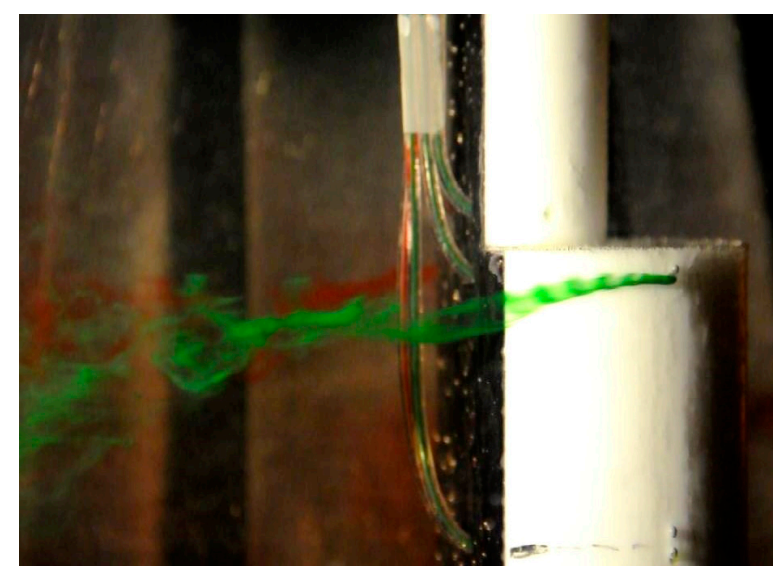

(b)

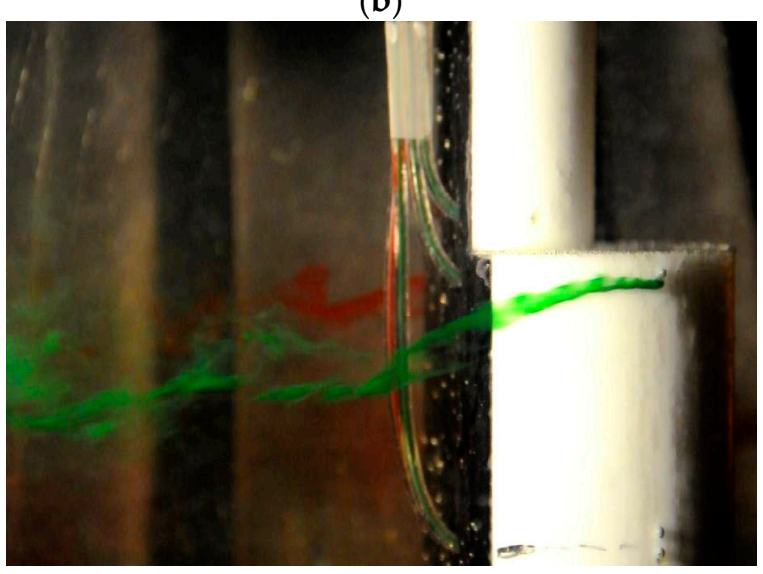

(c)

Figure 5. Wake structures around step cylinder at $z / D=-0.5 ;(\mathbf{a})[t=0]$; (b) $[t+1] ;(\mathbf{c})[t+1+1]$.

\subsection{Vortex Shedding}

The vortex shedding behind the cylinder cannot be described with averaged data. Therefore, Figure 6 shows an instant velocity field captured at the final time step of the simulation $(t=87.5 \mathrm{~s})$ for the horizontal planes in the center of the large cylinder, small cylinder, and at the cylinder step, respectively. As seen there is a clear shedding for all planes. The impacts of channel blockage, i.e., the ratio of the cylinder diameter ( $D$ or $d$ ) to channel width $(W)$ should be taken into consideration. The blockage ratio, $D / W$ and $d / W$, are 0.34 and 0.17 for large and small cylinders, respectively, in this study. 


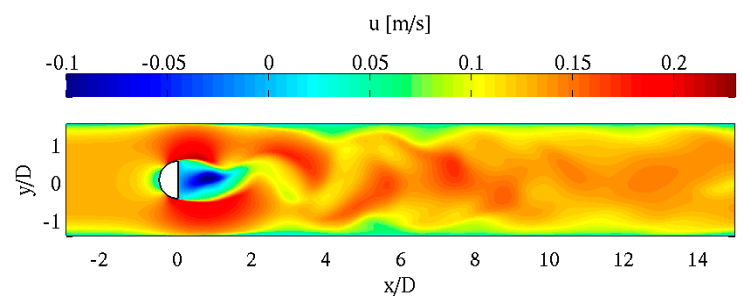

(a)

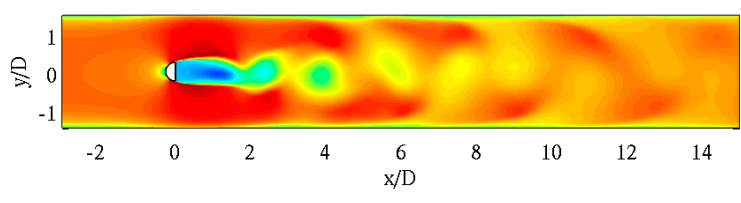

(b)

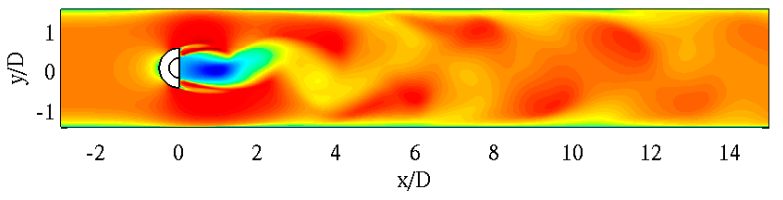

(c)

Figure 6. Streamwise velocity of the final time step $(t=87.5 \mathrm{~s})$ at (a) $z / D=-0.5 ;(\mathbf{b}) z / D=0.5$; and (c) $z / D=0.0$.

The shedding can also be studied in the vorticity field, for instance, Figure 7 shows the vertical vorticity component at one of the last time steps $(t=86.5 \mathrm{~s})$. Counter-rotating vortices can be seen for all three planes and in the plane at the step there seems to be some interaction between the vortices shed from the small and large cylinder. Visualization experiments were also carried out for $z / D=-0.5$ and $z / D=0.5$ to verify the vorticity obtained from CFD. Figure 7 depicts the comparison of these with $\mathrm{CFD}$ at a similar stage of vortex shedding and it is shown that there is a qualitative agreement between experiments and simulations.

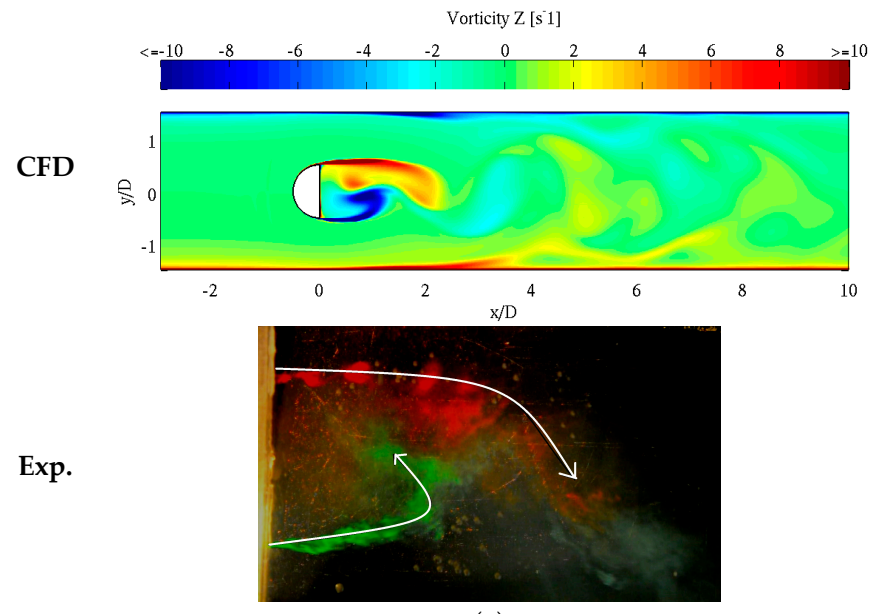

(a)

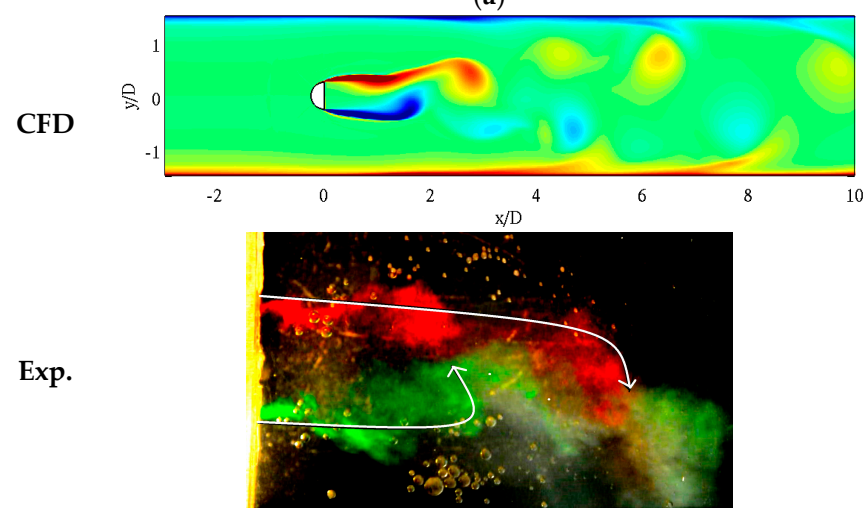

(b)

Figure 7. Cont. 


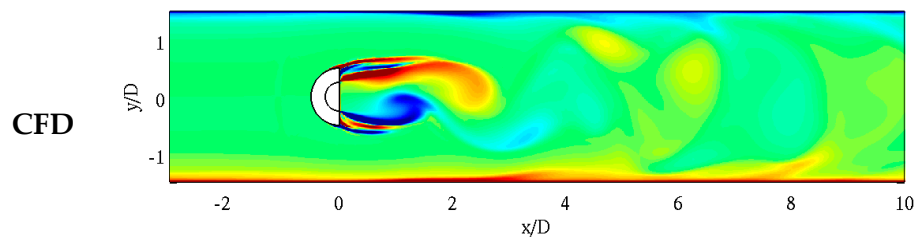

(c)

Figure 7. Vertical vorticity component $(t=86.5 \mathrm{~s})$ at (a) $z / D=-0.5 ;(\mathbf{b}) z / D=0.5$; and (c) $z / D=0.0$ along with the experimental visualization for $z / D=-0.5$ and $z / D=0.5$.

Another interesting variable is the fluid pressure. Figure 8 shows the pressure in the final time step at $z / D=-0.5$ and $z / D=0.5$. Zones where the pressure is lower than the surrounding pressure can be found throughout the vortex street, which could be of some importance when predicting and describing fish movement in this type of flow.

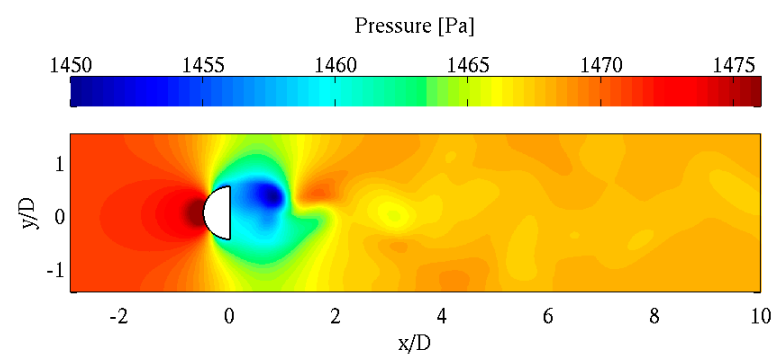

(a)

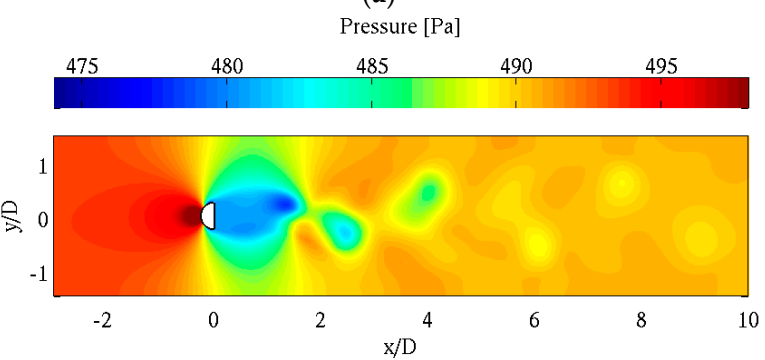

(b)

Figure 8. Fluid pressure at the final time step $(t=87.5 \mathrm{~s})$ at $(\mathbf{a}) z / D=-0.5$; and $(\mathbf{b}) z / D=0.5$.

The shedding frequency $(f)$ was determined from the velocity spectra using the fast Fourier transform (FFT). The concept of LDV is that measurements are carried out if a particle travels through the volume of measurement, meaning that often there is, no signal available [33-35]. Thus, sampling data from LDV are random offering challenges for the estimation of the power spectrum. Nobach $[29,31,36]$ described the details of up-to-date available methods to analyze the frequency spectrum from arbitrarily sampled data acquired by LDV measurements.

The estimation of power spectra presented in this paper is the interpolation of the arbitrarily or randomly sampled LDV data to obtain a uniform velocity over time, which is then re-sampled in equal steps with a known sampling frequency close to the average sampling frequency. The mean velocity for each signal was then subtracted as the fluctuations around the mean $u^{\prime}$ describing the periodicity. The dominant peak in each spectrum is then assumed to correspond to the vortex shedding frequency $(f)$.

The shedding frequency for the large cylinder and step are evaluated at $x / D=2.5, y / D=0.75$ and the shedding frequency from the small cylinder at $x / d=2.5$ and $y / d=0.75$, see Figure 9 . For the shedding behind the large cylinder the dominating peak gives $f=0.34 \mathrm{~Hz}$ for the experiments and $f=0.38 \mathrm{~Hz}$ for the simulations corresponding to Strouhal numbers $S t_{\mathrm{LDV}}=f D / U_{\infty} \approx 0.234$ and 
$S t_{\mathrm{CFD}} \approx 0.262$ which can be considered to be in good agreement. For the shedding behind the smaller cylinder the simulation gives the same frequency as behind the large cylinder whereas the LDV gives a peak at $f=0.6 \mathrm{~Hz}$ which is more consistent with other studies [37-39] behind fully-circular stepped cylinders. For the shedding behind the step, the experiments yield two dominant peaks, one at $f=0.36 \mathrm{~Hz}$, which should correspond to the shedding from the large cylinder and $f=0.6 \mathrm{~Hz}$ which corresponds to the shedding from the small one, whereas the simulation once again gives the same frequency as for the large cylinder, not capturing the dynamics of the small cylinder or the step. This may be traced to the SST turbulence model's inability to capture the shedding correctly; other possible contributors could be that the time-step is too large during the experiments so that some spatial or temporal details are neglected or that the flow at the smaller cylinder is not yet fully developed even after 3500 iterations. In addition, the effect of blockage on vortex shedding for the flow past a step cylinder can also be significant. Some previous studies $[37,38,40-43]$ have shown that the effect of channel blockage on vortex shedding for the flow past a step cylinder can also be significant as the channel blockage affects $f$, consequently influencing St. The increase of shedding frequency with the increase of the blockage ratios is evidently observed in these spectral results (Figure 6). Therefore, channel blockage could also be the reason on varying the shedding frequency behind step cylinder (large and small cylinders).
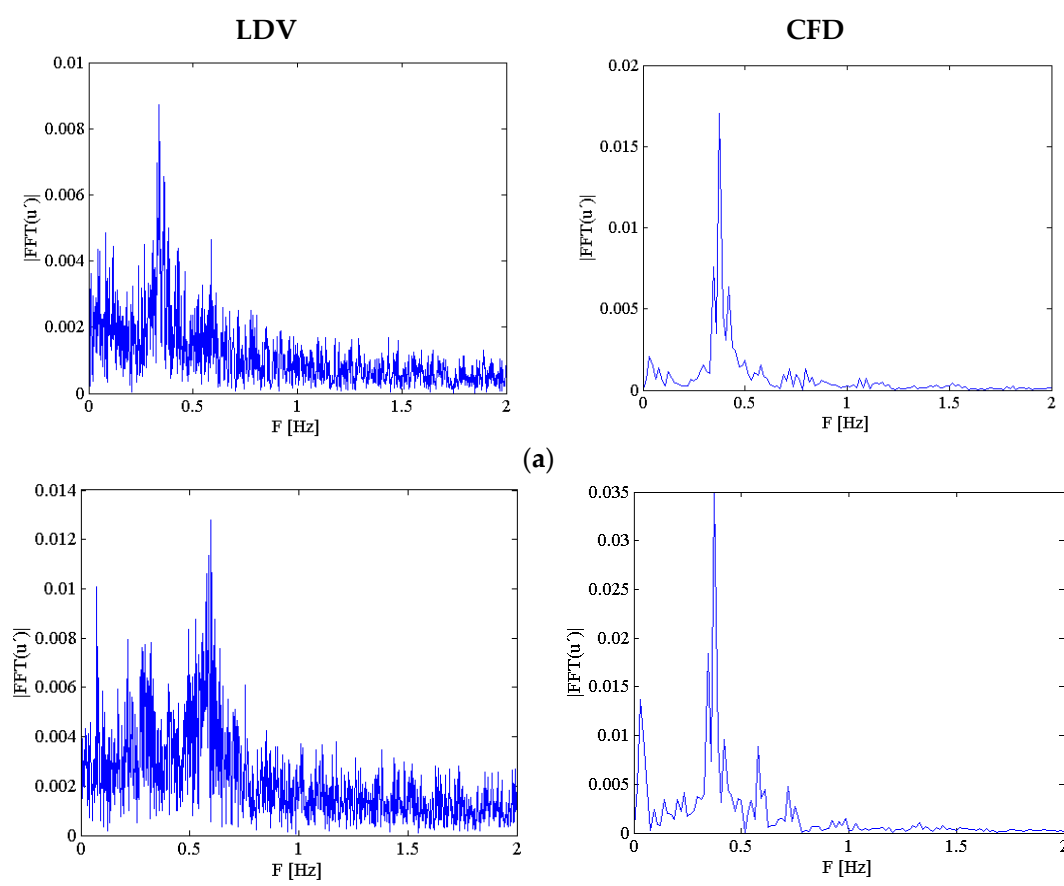

(a)
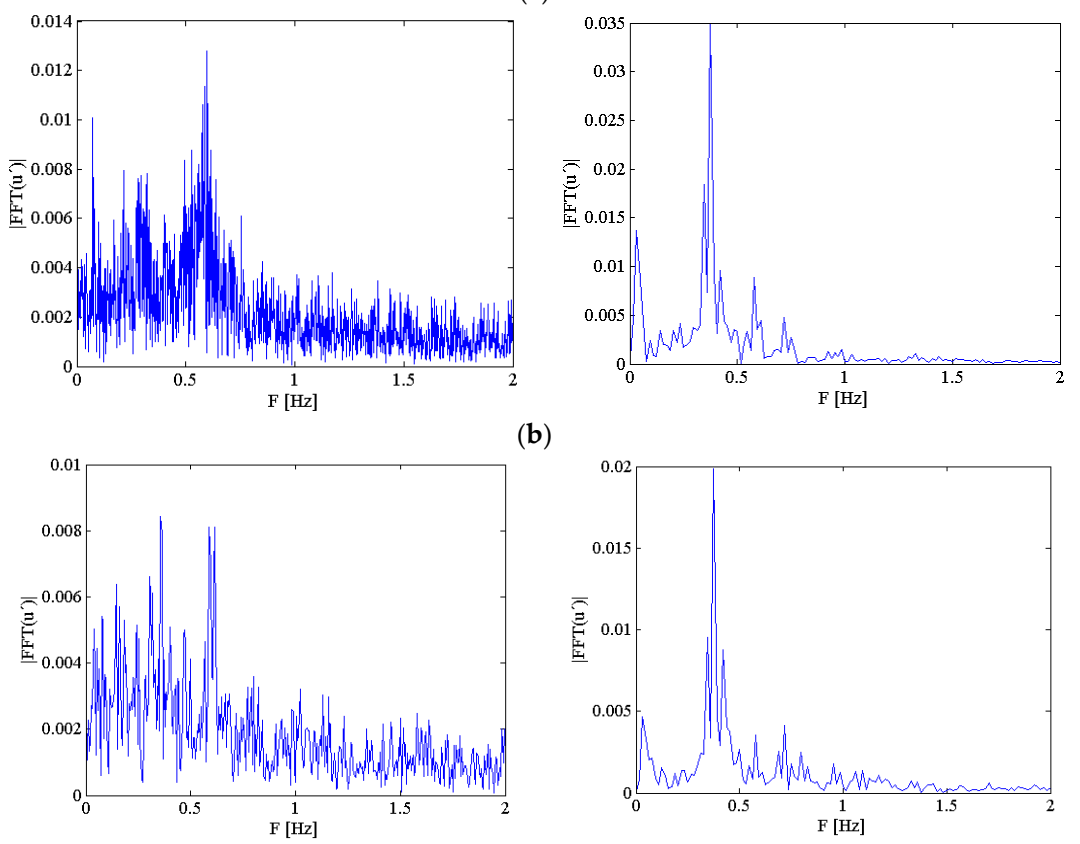

(b)

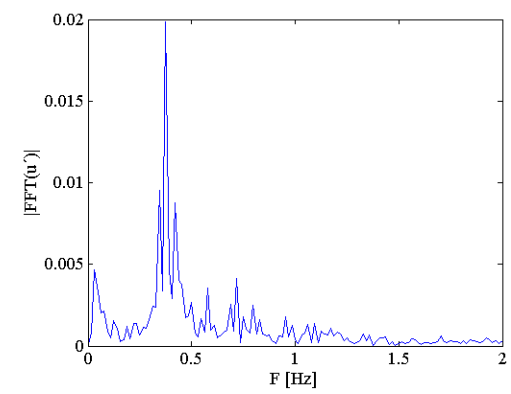

(c)

Figure 9. Single-sided FFT amplitude spectrum of the streamwise velocity fluctuations for LDV and CFD at (a) the large cylinder, $x / D=2.5, z / D=-0.5, y / D=0.75$ (b) small cylinder, $x / d=2.5, z / D=0.5$, $y / d=0.75$; and (c) diameter step, $x / D=2.5, z / D=0.0, y / D=0.75$. 


\section{Concluding Remarks}

An investigation with LDV measurements and CFD simulations behind a semi-circular cylinder with a step has been conducted. Additional flow visualization was also carried out to explore the wake structure. A summary of key features of the wake flows obtained in this study is given below:

1. The main result is that the measured vortex shedding frequency is, $f=0.34 \mathrm{~Hz}$ behind the large cylinder and $f=0.6 \mathrm{~Hz}$ behind the small cylinder, while it has two dominating frequencies across the step, $f=0.36 \mathrm{~Hz}$ and $f=0.6 \mathrm{~Hz}$. This implies that a step cylinder can be used to attract fish of different sizes and facilitate their motion up-stream the fishway.

2. Experiments yield that the wake length is longer along the step $(z / D=0.0)$ and it reduces for the large cylinder $(z / D=-0.5)$, as well as the small cylinder $(z / D=0.5)$. The simulation over-predicts all of these wake lengths.

3. Flow visualization finds the development of three-dimensional (3D) flow structures along with well-known Von Karman vortex street.

4. Simulation results show acceptable agreement with the experimental measurements behind the large cylinder, but fail to capture the dynamics of the small cylinder diameter.

Future studies with tomographic-PIV would enable the detailed vortex dynamics of the flow behind the stepped half-cylinders to be investigated. Additional simulations with more advanced models, such as the Reynolds stress models and large eddy simulations, could also provide additional insights into this type of problem.

For the application of fish migration, it would also be interesting to test these configurations on fish in laboratory studies, as well as in the field, to see how different species and sizes of fish respond to different geometries and flow conditions.

Acknowledgments: This work has been funded by the collaboration initiative StandUp for Energy. The research program is a part of the Swedish government's commitment to high quality research in areas of strategic importance.

Author Contributions: S. M. Sayeed-Bin-Asad, Tord Staffan Lundström and Anders Gustav Andersson conceived and designed the experiments; S. M. Sayeed-Bin-Asad performed the experiments and analyzed the data; Anders Gustav Andersson performed the simulation; S. M. Sayeed-Bin-Asad wrote the paper; All authors read and approved the final manuscript.

Conflicts of Interest: The authors declare no conflict of interest.

\section{References}

1. Swedenergy. Svensk Energi-Swedenergy-AB. Available online: http://www.svenskenergi.se/ (accessed on 12 October 2016).

2. Kaveh, A. Experimental Investigation of a Kaplan Runner under Steady-State and Transient Operations. Ph.D. Thesis, Luleå University of Technology, Luleå, Sweden, 2016.

3. Liao, J.C.; Beal, D.N.; Lauder, G.V.; Triantafyllou, M.S. Fish exploiting vortices decrease muscle activity. Science 2003, 302, 1566-1569. [CrossRef] [PubMed]

4. Liao, J.C. A review of fish swimming mechanics and behaviour in altered flows. Philos. Trans. R. Soc. B Biol. Sci. 2007, 362, 1973-1993. [CrossRef] [PubMed]

5. Stewart, W.J.; Tian, F.B.; Akanyeti, O.; Walker, C.J.; Liao, J.C. Refuging rainbow trout selectively exploit flows behind tandem cylinders. J. Exp. Biol. 2016, 219, 2182-2191. [CrossRef] [PubMed]

6. Akanyeti, O.; Thornycroft, P.J.M.; Lauder, G.V.; Yanagitsuru, Y.R.; Peterson, A.N.; Liao, J.C. Fish optimize sensing and respiration during undulatory swimming. Nat. Commun. 2016, 7, 11044. [CrossRef] [PubMed]

7. Sayeed-Bin-Asad, S. Velocity Distribution Measurements in A Fishway Like Open Channel by Laser Doppler Anemometry (LDA). EPJ Web. Conf. 2016. [CrossRef]

8. Liao, J.C.; Beal, D.N.; Lauder, G.V.; Triantafyllou, M.S. The Kármán gait: Novel body kinematics of rainbow trout swimming in a vortex street. J. Exp. Biol. 2003, 206, 1059-1073. [CrossRef] [PubMed]

9. Dunn, W.; Tavoularis, S. Vortex shedding from a step-cylinder in spanwise sheared flow. Phys. Fluids 2011, 23, 035109. [CrossRef] 
10. Dunn, W.; Tavoularis, S. Experimental studies of vortices shed from cylinders with a step-change in diameter. J. Fluid Mech. 2006, 555, 409-437. [CrossRef]

11. Morton, C.; Yarusevych, S. Vortex shedding in the wake of a step cylinder. Phys. Fluids 2010, $22,083602$. [CrossRef]

12. Morton, C.; Yarusevych, S. Three-dimensional flow and surface visualization using hydrogen bubble technique. J. Vis. 2015, 18, 47-58.

13. Morton, C.; Yarusevych, S. On vortex shedding from low aspect ratio dual step cylinders. J. Fluids Struct. 2014, 44, 251-269. [CrossRef]

14. Morton, C.; Yarusevych, S. Vortex dynamics in the turbulent wake of a single step cylinder. J. Fluids Eng. 2014, 136, 031204. [CrossRef]

15. McClure, J.; Morton, C.; Yarusevych, S. Flow development and structural loading on dual step cylinders in laminar shedding regime. Phys. Fluids 2015, 27, 063602. [CrossRef]

16. Morton, C.R.; Yarusevych, S. A combined experimental and numerical study of flow past a single step cylinder. In Proceedings of the ASME 2010 3rd Joint US-European Fluids Engineering Summer Meeting Collocated with 8th International Conference on Nanochannels, Microchannels, and Minichannels, Montreal, QC, Canada, 1-5 August 2010; American Society of Mechanical Engineers: New York, NY, USA, 2010.

17. Morton, C.; Yarusevych, S.; Scarano, F. A tomographic particle image velocimetry investigation of the flow development over dual step cylinders. Phys. Fluids 2016, 28, 025104. [CrossRef]

18. Rafati, S. Investigating Step Cylinder Wake Topology with Tomographic PIV; Delft University of Technology: Delft, The Netherlands, 2014.

19. Ko, N.; Leung, W.; Au, H. Flow behind two coaxial circular cylinders. J. Fluids Eng. 1982, 104, $223-227$. [CrossRef]

20. Yagita, M.; Kojima, Y.; Matsuzaki, K. On vortex shedding from circular cylinder with step. Bull. JSME 1984, 27, 426-431. [CrossRef]

21. Lewis, C.; Gharib, M. An exploration of the wake three dimensionalities caused by a local discontinuity in cylinder diameter. Phys. Fluids A Fluid Dyn. 1992, 4, 104-117. [CrossRef]

22. Norberg, C. An experimental study of the flow around cylinders joined with a step in diameter. In Proceedings of the 11th Australasian Fluid Mechanics Conference, Hobart, Australia, 14-18 December 1992.

23. Ko, W.-M.; Chan, S.-K. Pressure Distributions on Circular Cylinders with Stepwise Change of the Diameter; American Society of Mechanical Engineers: New York, NY, USA, 1984.

24. Ko, N.; Chan, A. In the intermixing region behind circular cylinders with stepwise change of the diameter. Exp. Fluids 1990, 9, 213-221. [CrossRef]

25. Ko, N.; Chan, A. Wakes behind circular cylinders with stepwise change of diameter. Exp. Therm. Fluid Sci. 1992, 5, 182-187. [CrossRef]

26. Chua, L.; Liu, C.; Chan, W. Measurements of a step cylinder. Int. Commun. Heat Mass Transf. 1998, 25, 205-215. [CrossRef]

27. Morton, C.; Yarusevych, S. Modeling Flow over a Circular Cylinder with a Stepwise Discontinuity. In Proceedings of the 39th AIAA Fluid Dynamics Conference, San Antonio, TX, USA, 22-25 June 2009.

28. Morton, C.; Yarusevych, S. Cross flow over cylinders with two stepwise discontinuities in diameter. In Proceedings of the Turbulence and Shear Flow Phenomena Conference, Ottawa, ON, Canada, 28-30 July 2011.

29. Nobach, H. Present Methods to Estimate the Cross-Correlation and Cross-Spectral Density for Two-Channel Laser Doppler Anemometry. In Proceedings of the 18th International Symposium on the Application of Laser and Imaging Techniques to Fluid Mechanics, Lisbon, Portugal, 4-7 July 2016.

30. Norberg, C. LDV-Measurements in the Near Wake of a Circular Cylinder. Available online: http:/ / www.ht. energy.lth.se/fileadmin/ht/Norberg-FEDSM98-5202.pdf (accessed on 12 October 2016).

31. Hirt, C.W.; Nichols, B.D. Volume of fluid (VOF) method for the dynamics of free boundaries. J. Comput. Phys. 1981, 39, 201-225. [CrossRef]

32. Menter, F.R. Review of the shear-stress transport turbulence model experience from an industrial perspective. Int. J. Comput. Fluid Dyn. 2009, 23, 305-316. [CrossRef]

33. Morton, C.; Yarusevych, S. An experimental investigation of flow past a dual step cylinder. Exp. Fluids 2012, 52, 69-83. [CrossRef] 
34. Velte, C.M.; George, W.K.; Buchhave, P. Estimation of burst-mode LDA power spectra. Exp. Fluids 2014, 55, 1-20. [CrossRef]

35. Müller, E.; Nobach, H.; Tropea, C. A refined reconstruction-based correlation estimator for two-channel, non-coincidence laser Doppler anemometry. Meas. Sci. Technol. 1998, 9, 442. [CrossRef]

36. Nobach, H. Laser Doppler Data Processing. Available online: http://ldvproc.nambis.de/programs/pyLDV. html (accessed on 10 October 2016).

37. Turki, S.; Abbassi, H.; Nasrallah, S.B. Effect of the blockage ratio on the flow in a channel with a built-in square cylinder. Comput. Mech. 2003, 33, 22-29. [CrossRef]

38. Zong, L.; Nepf, H. Vortex development behind a finite porous obstruction in a channel. J. Fluid Mech. 2012, 691, 368-391. [CrossRef]

39. Morton, C.R. Experimental and Numerical Investigations of the Flow Development over Circular Cylinders with Stepwise Discontinuities in Diameter. Master's Thesis, University of Waterloo, Waterloo, ON, Canada, 2010.

40. Sahin, M.; Owens, R.G. A numerical investigation of wall effects up to high blockage ratios on two-dimensional flow past a confined circular cylinder. Phys. Fluids (1994-Present) 2004, 16, 1305-1320. [CrossRef]

41. Mettu, S.; Verma, N.; Chhabra, R. Momentum and heat transfer from an asymmetrically confined circular cylinder in a plane channel. Heat Mass Transf. 2006, 42, 1037-1048. [CrossRef]

42. Okajima, A.; Yi, D.; Sakuda, A.; Nakano, T. Numerical study of blockage effects on aerodynamic characteristics of an oscillating rectangular cylinder. J. Wind Eng. Ind. Aerodyn. 1997, 67, 91-102. [CrossRef]

43. Park, C.-W.; Lee, S.-J. Free end effects on the near wake flow structure behind a finite circular cylinder. J. Wind Eng. Ind. Aerodyn. 2000, 88, 231-246. [CrossRef]

(C) 2017 by the authors. Licensee MDPI, Basel, Switzerland. This article is an open access article distributed under the terms and conditions of the Creative Commons Attribution (CC BY) license (http:/ / creativecommons.org/licenses/by/4.0/). 